Article

\title{
Serum Anti-Müllerian Hormone in the Diagnosis of Polycystic Ovary Syndrome in Association with Clinical Symptoms
}

\author{
Nada Ahmed ${ }^{1}$, Asma A. Batarfi ${ }^{1}$, Osama S. Bajouh ${ }^{2,3}$ and Sherin Bakhashab ${ }^{1,3, * \mathbb{C}}$ \\ 1 Biochemistry Department, King Abdulaziz University, P.O. Box 80218, Jeddah 21589, Saudi Arabia; \\ nahmed0028@stu.kau.edu.sa (N.A.); asma.batarfi@hotmail.com (A.A.B.) \\ 2 Department of Obstetrics and Gynecology, Faculty of Medicine, King Abdulaziz University, P.O. Box 80205, \\ Jeddah 21589, Saudi Arabia; dr_bajouh@yahoo.com \\ 3 Center of Innovation in Personalized Medicine, King Abdulaziz University, P.O. Box 80216, Jeddah 21589, \\ Saudi Arabia \\ * Correspondence: sbakhashab@kau.edu.sa; Tel.: +966-126-400-000
}

Received: 6 September 2019; Accepted: 28 September 2019; Published: 1 October 2019

\begin{abstract}
Polycystic ovary syndrome (PCOS) is one of the most prevalent endocrine diseases affecting women of reproductive age. The pathogeny of PCOS is still not completely understood, but one contributing factor that has been proposed is anti-Müllerian hormone (AMH). There is currently no clear correlation between levels of AMH and incidence of PCOS in Saudi Arabian patients. The goal of this study was to determine the threshold of AMH and correlate it with PCOS clinical features to facilitate a proper diagnosis for PCOS. In this case-control study, we recruited 79 PCOS women and 69 normal ovulatory women; PCOS patients were diagnosed according to the Rotterdam criterion. On days $2-4$ of the menstrual cycle, transvaginal/abdominal ultrasound was performed and serum levels of $\mathrm{AMH}$, luteinizing hormone (LH), and follicle-stimulating hormone (FSH) were measured for all participants. The receiver operating characteristic curve (ROC) was used to determine the AMH diagnostic cut-off at $3.19 \mathrm{ng} / \mathrm{mL}$, with $72 \%$ sensitivity and $70 \%$ specificity; AMH $>3.19 \mathrm{ng} / \mathrm{mL}$ was significantly correlated with PCOS. High AMH levels were correlated with age at menarche, polycystic ovarian morphology (PCOM), and oligo/amenorrhea. Serum AMH is a promising diagnostic marker of ovarian dysfunction in PCOS patients especially in cases in which the evaluation of PCOM was complicated.
\end{abstract}

Keywords: polycystic ovary syndrome; anti-Müllerian hormone; oligo/amenorrhea; hyperandrogenism

\section{Introduction}

Polycystic ovary syndrome (PCOS), a multifactorial syndrome, is one of the most common endocrine diseases affecting reproductive-aged women, with an incidence rate of 9-18\% [1]. Currently, it is diagnosed according to the Rotterdam criterion that defines PCOS by the presence of two out of three of the following symptoms: polycystic ovarian morphology (PCOM), clinical or biochemical hyperandrogenism (HA) and oligo/amenorrhea (OA) [2,3]. PCOM is diagnosed by the presence of at least 12 follicles 2-9 $\mathrm{mm}$ in diameter, and measured using transvaginal or abdominal ultrasound [2]. Ethnic and racial differences in follicle number per ovary and ovarian volume in determining PCOM diagnostic cut-offs have been measured in Chinese and Turkish women [4,5].

Recent research concerning different aspects of PCOS has produced new insights into assessment and treatment of the disorder. The relationship between PCOS and anti-Müllerian hormone (AMH) is of interest as AMH plays an important role in ovarian function. It preserves the follicles in the primordial stage and estimates the number of ova in the ovaries, thereby providing an indication of 
the ovarian reserve [6,7]. Previous studies have shown that serum AMH levels correspond with the number of antral follicles which are augmented in PCOS and PCOM [8,9]. Women with PCOS were found to have higher serum AMH due to a greater number of antral follicles and greater production of AMH per follicle [10,11], making AMH possibly an important factor in the diagnosis of PCOS. Dewailly et al. [12] proposed AMH levels greater than $5 \mathrm{ng} / \mathrm{mL}$ could be a diagnostic substitution for PCOM, while another study suggested AMH levels greater than $3.8 \mathrm{ng} / \mathrm{mL}$ [13]. The presence of two out of three clinical features (OA, HA, and/or AMH) was found to have $96 \%$ sensitivity and $100 \%$ specificity among patients previously diagnosed with PCOS, according to the Rotterdam criterion [13]. Moreover, Dewailly et al. [14] developed merged PCOS diagnosis criteria using Rotterdam and non-Rotterdam definitions, proposing an excessive follicle number or serum AMH concentration as a surrogate marker for the diagnosis of PCOS in the absence of either OA or HA [14]. Previous studies have shown the potency of serum AMH as a diagnostic marker for PCOS and in determining the optimum diagnostic level $[9,12]$. Furthermore, a pregnant mouse model treated with high AMH during pregnancy caused changes in the fetus, which ended up having a PCOS-like disorder in adulthood [15]. Ethnicity has been associated with variable AMH levels, and Asian women have lower AMH at a given age than Caucasians [16]. The AMH diagnostic cut-off has been demonstrated at $4.7-5 \mathrm{ng} / \mathrm{mL}$ in Caucasians [17], and $10 \mathrm{ng} / \mathrm{mL}$ in Japanese and Korean women $[18,19]$. In this study, we aim to determine the PCOS diagnostic cut-off for AMH in the Saudi population. We also study the relationship between the serum AMH threshold in patients and prominent clinical parameters of PCOS. This will allow for assessment of the diagnostic accuracy of using AMH as a surrogate compared to the Rotterdam criterion, and may contribute to the proper diagnosis of PCOS among Saudi women.

\section{Materials and Methods}

\subsection{Study Design}

This was a case-control, observational, and randomized study implemented in the Obstetrics and Gynecology Clinics, King Abdulaziz University Hospital, Jeddah, Saudi Arabia and Center of Innovation in Personalized Medicine (CIPM), KAU, Jeddah, Saudi Arabia, between 2016-2018. The clinical study, sample size selection, and exclusion criteria have been previously discussed [20]. Using Raosoft (www.raosoft.com), the appropriate sample size was calculated to be 196 women of reproductive age between 18 and 38 years old. The study was approved by the Biomedical Ethics Unit, Faculty of Medicine, KAU (approval number: 407-15) on 19 January 2016 and written informed consent was obtained from participants prior to sample collection. The study was conducted in accordance with the Declaration of Helsinki.

The case group was composed of 98 selected PCOS patients diagnosed based on the Rotterdam Criteria Consensus, and the control group was composed of 98 women with normal ovulation. The enrolled women were not under medication. On days 2-4 of the menstrual cycle, transvaginal or abdominal ultrasound (SonixTouch, Ultrasonix Medical Corporation, Richmond, BC, Canada) were performed, and serum hormonal levels for luteinizing hormone (LH) and follicle-stimulating hormone (FSH) were measured with an automated multi-analysis system using electrochemiluminescence immunoassay kits (Roche, Basel, Switzerland). Serum AMH levels were determined by an enzyme-linked immunosorbent assay using an Ultra-Sensitive Anti-Müllerian hormone/Müllerian inhibiting substance Kit (AnshLabs, Webster, TX, USA) according to the manufacturer's instructions.

HA was assessed clinically through the presence of hirsutism, androgenic alopecia, and acne. Patients' hirsutism was evaluated by a Ferriman-Gallwey score greater than 8. OA was defined as less than eight menstrual cycles in 12 months, or if the menstrual interval was more than 35 days. Using transvaginal or abdominal ultrasound, PCOM was identified by the presence of at least 12 follicles measuring $2-9 \mathrm{~mm}$ in diameter in one ovary [2]. Poor quality samples were excluded from the statistical analyses, resulting in 79 cases and 69 controls. 
Initially, patients were diagnosed according to the Rotterdam criterion. After the selection of the AMH threshold value at $3.19 \mathrm{ng} / \mathrm{mL}$, the PCOS group was re-evaluated twice. We used two different classification methods. The first classification identified PCOS as exhibiting two out of three features by which AMH may replace PCOM: OA, HA, or AMH > 3.19. The second classification defined PCOS s having two out PCOM, HA, or AMH > 3.19. After that, two new receiver operating characteristic (ROC) curves (one for each classification) were constructed to assess the sensitivity and specificity of a $3.19 \mathrm{ng} / \mathrm{mL}$ AMH diagnostic cut-off.

\subsection{Statistical Analysis}

A normality test was performed to determine if the data were normally distributed. Data are presented as (median \pm interquartile range (IQR)), $p$-values were calculated using a Mann-Whitney test. The ROC curve was utilized to examine the diagnostic cut-off of AMH. The chi-squared test was used to demonstrate the correlation between AMH, PCOS, and PCOS variables. In the PCOS group, the association was evaluated using binary logistic regression or multinomial logistic regression between AMH cut-off and PCOS clinical features. Statistical analyses were performed using IBM SPSS software version 24.0 (SPSS Inc., Armonk, NY, USA). A $p$-value $<0.05$ was considered statistically significant.

\section{Results}

Clinical characteristics are summarized in Table 1 . The case and control groups were matched for age ( $23 \pm 9$ vs $21 \pm 3.5, p=0.067)$ but not on body mass index (BMI, $25.1 \pm 6.98 \mathrm{~kg} / \mathrm{m}^{2}$ vs $23 \pm 5.94$ $\left.\mathrm{kg} / \mathrm{m}^{2}, p=0.005\right)$. The serum levels of AMH and LH were significantly higher in the case group than in the controls $(4.8 \pm 4.76 \mathrm{ng} / \mathrm{mL}$ vs $2.3 \pm 1.41 \mathrm{ng} / \mathrm{mL}, p<0.0001$; and $8.4 \pm 8.55 \mathrm{IU} / \mathrm{mL}$ vs $5.8 \pm 5.2$ $\mathrm{IU} / \mathrm{mL}, p=0.003$, respectively). Although there was no significant difference in serum levels of FSH, the $\mathrm{LH} / \mathrm{FSH}$ ratio was significantly higher amongst the case group compared to the control group (1.7 \pm 1.79 vs $1.2 \pm 1.47, p=0.006)$. In the PCOS group, 73 women had PCOM $(92 \%), 66$ women had AO $(83 \%)$, and 55 women had symptoms of HA (69.6\%).

Table 1. A summary of the clinical characteristics of polycystic ovary syndrome (PCOS) patients and control subjects.

\begin{tabular}{cccc}
\hline Variable & Control $(n=69)$ & PCOS $(n=79)$ & $p$-Value \\
\hline Age (years) & $21 \pm 3.5$ & $23 \pm 9$ & 0.067 \\
BMI (kg/m $\left.{ }^{2}\right)$ & $23 \pm 5.94$ & $25.1 \pm 6.98$ & $0.005^{* *}$ \\
LH (IU/L) & $5.8 \pm 5.2$ & $8.4 \pm 8.55$ & $0.003^{* *}$ \\
FSH (IU/L) & $4.6 \pm 2.75$ & $4.7 \pm 2.45$ & 0.618 \\
LH/FSH ratio & $1.2 \pm 1.47$ & $1.7 \pm 1.79$ & $0.006^{* *}$ \\
AMH (ng/mL) & $2.3 \pm 1.41$ & $4.8 \pm 4.76$ & $<0.0001^{* * *}$ \\
\hline
\end{tabular}

Values are expressed as median \pm interquartile range (IQR), and $p$-values were calculated using a Mann-Whitney test for data with non-normal distribution. $p$-value $<0.05$ is statistically significant. BMI: body mass index; LH: luteinizing hormone; FSH: follicle-stimulating hormone; AMH: anti-Müllerian hormone. ${ }^{* *} p<0.01,{ }^{* * *} p<0.001$.

To determine the AMH diagnostic cut-off, the ROC curve was applied (Figure 1). A $3.19 \mathrm{ng} / \mathrm{mL}$ AMH cut-off level was determined with $72 \%$ sensitivity, $70 \%$ specificity, and the area under the curve equaling $0.785(0.713-0.858)$, with a $95 \%$ confidence interval. Greater than $3.19 \mathrm{ng} / \mathrm{mL}$ was determined to be significantly associated with PCOS $(p<0.001)$ using the chi-squared test. 


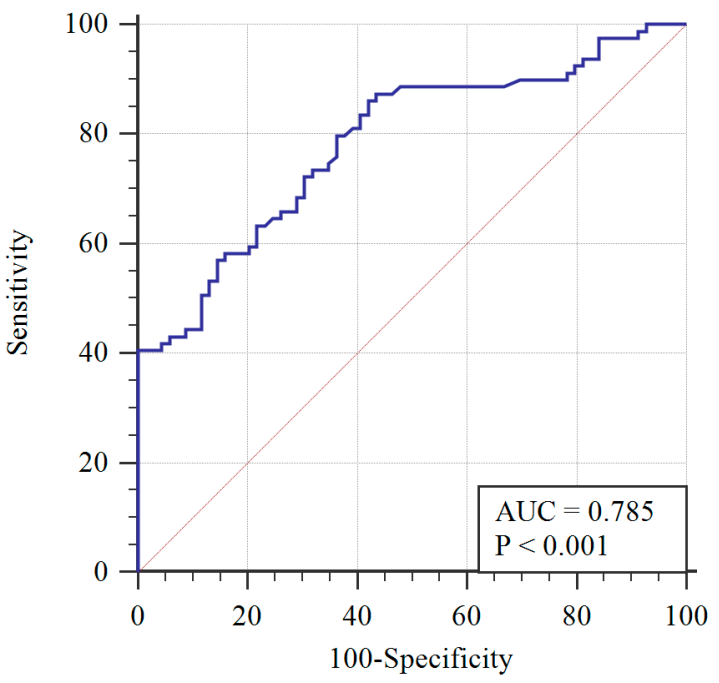

Figure 1. Receiver operating characteristic (ROC) curve of AMH with respect to PCOS diagnosis (blue line) and reference curve (red line). AUC: Area under the curve.

Pearson's correlation analysis revealed a positive correlation between AMH and age at menarche $(r=0.324, p=0.018)$ and no correlation between AMH and BMI, LH, FSH, and LH/FSH ratio (Table 2).

Table 2. Associations between AMH cut-off and PCOS scale factors.

\begin{tabular}{ccc}
\hline Clinical Characteristics & $r$ & $p$-Value \\
\hline Age (years) & -0.113 & 0.328 \\
Age at menarche (years) & 0.324 & $0.018^{*}$ \\
BMI $\left(\mathrm{kg} / \mathrm{m}^{2}\right)$ & -0.037 & 0.750 \\
LH (IU/L) & 0.031 & 0.794 \\
FSH (IU/L) & 0.019 & 0.870 \\
LH/FSH ratio & 0.073 & 0.540
\end{tabular}

$p$-values were calculated by Pearson's correlation test and Pearson's correlation coefficient $r$ is used to present the associations. $p$-values $<0.05$ are statistically significant. BMI: Body mass index; LH: luteinizing hormone; FSH: Follicle-stimulating hormone. ${ }^{*} p<0.05$.

Additionally, a chi-squared test was performed on the clinical features of PCOS and AMH cut-off levels in the case group. There was a significant association (Table 3) between AMH at the threshold cut-off with two variables, PCOM $(p=0.048)$ and OA $(p=0.015)$, whereas no association was detected with HA $(p=1.000)$.

Table 3. Association between PCOS clinical symptoms and AMH at threshold cut-off level.

\begin{tabular}{cccc}
\hline Clinical Symptoms & AMH $\geq \mathbf{3 . 1 9}$ & AMH $<3.19$ & $p$-Value \\
\hline PCOM (Yes/No) & $55 / 2$ & $18 / 4$ & 0.048 \\
OA (Yes/No) & $44 / 13$ & $22 / 0$ & 0.015 \\
HA (Yes/No) & $39 / 13$ & $16 / 6$ & 1.000 \\
\hline
\end{tabular}

$p$-values were calculated by a chi-squared test. $p$-values $<0.05$ are statistically significant. PCOM: Polycystic ovarian morphology; OA: Oligo/amenorrhea is defined by less than eight periods per year or a period length > 35 days; HA: Hyperandrogenism is defined by the presence of hirsutism, acne, or androgenic alopecia.

Logistic regression analysis performed between $\mathrm{AMH} \geq 3.19$ and $\mathrm{PCOM}$ or OA revealed a significant correlation with PCOM (odds ratio $(\mathrm{OR})=6.1, \mathrm{~B}=1.8, p=0.046$ ) and no correlation with OA. Moreover, multinomial logistic regression also showed a positive correlation between $\mathrm{AMH} \geq$ 3.19 and age at menarche (OR $=1.8, \mathrm{~B}=0.571, p=0.030)$. In other words, women with PCOS who experienced menarche at an older age were 1.8 times more likely to have higher $\mathrm{AMH}$. 
After re-assessing the case group using the AMH $\geq 3.19$ cut-off, the PCOM was substituted with AMH (first classification) to give the following distribution of patients: 73 still PCOS, and 6 non-PCOS. The AMH cut-off was re-calculated, and a sensitivity of $78 \%$ and specificity of $100 \%$ were obtained at $\mathrm{AMH}=3.19 \mathrm{ng} / \mathrm{mL}$ (Figure 2). When AMH substituted OA, the PCOS group included 70 patients, while the non-PCOS group included 9. An AMH cut-off greater than 3.19 was found to have $81 \%$ sensitivity and 100\% specificity (Figure 3).

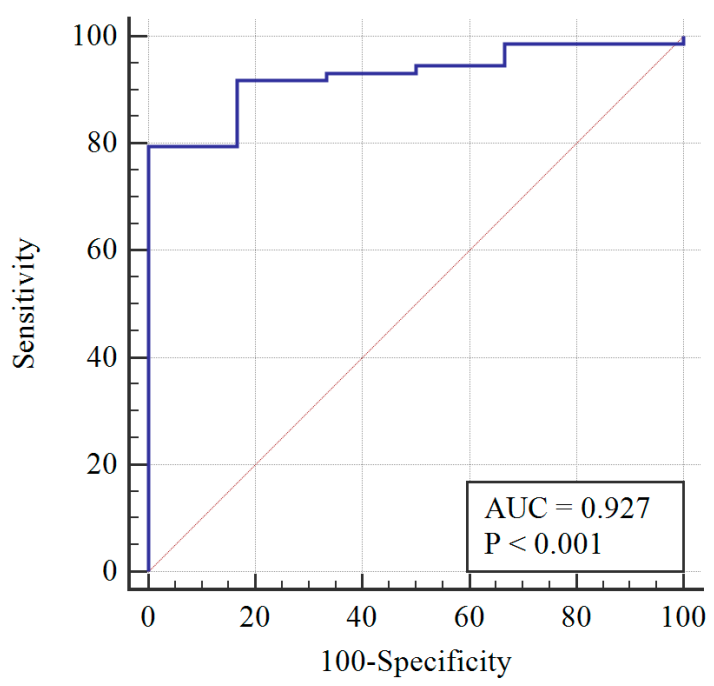

Figure 2. Diagnosis capability of OA, HA, or AMH > $3.19 \mathrm{ng} / \mathrm{mL}$ across the PCOS group (blue line) and reference curve (red line). AUC: Area under the curve.

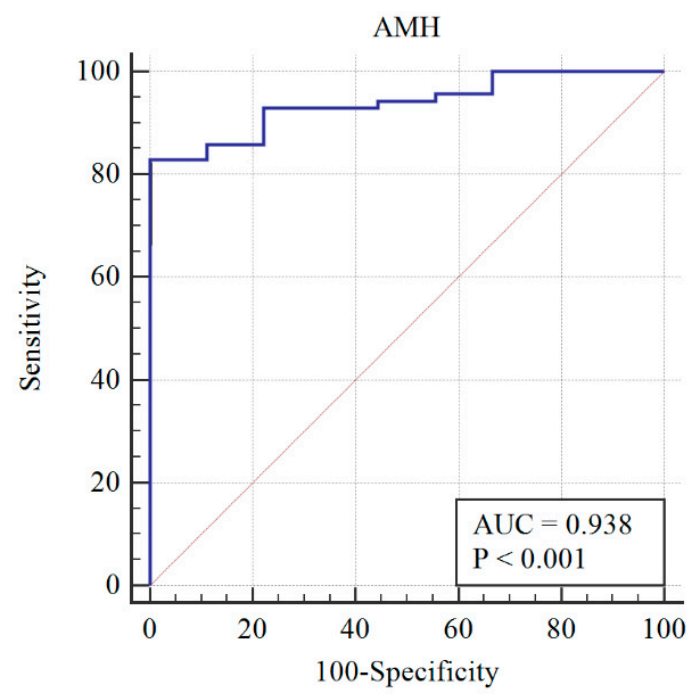

Figure 3. Diagnosis capability of PCOM, HA, or AMH $>3.19 \mathrm{ng} / \mathrm{mL}$ across the PCOS group (blue line) and reference curve (red line). AUC: Area under the curve.

\section{Discussion}

The etiology of PCOS is still unclear and the ability to make an integrated diagnosis among clinicians is difficult due to subjective phenotypes. Furthermore, it is well established that PCOS is associated with insulin resistance. If this fact is combined with delayed or confused PCOS diagnosis, then the prognosis of type 2 diabetes and cardiovascular disease may be accelerated [21-23]. Moreover, the evaluation of PCOM through abdominal ultrasound can be difficult, especially in virgins or obese women. Thus, easier, clearer, and more quantitative diagnostic criteria are essential. Serum AMH can now function within the diagnostic criteria, as serum AMH level has not only shown a significant 
association with PCOS, but can also reflect the severity of the disease [24,25]. Moreover, it can be easily measured at any time during a woman's cycle [26].

In this study of a sample among the Saudi population, we assessed whether serum AMH was a possible additional tool for the diagnosis of PCOS regardless of patient phenotype. Our results showed a significant difference in AMH levels among the control and PCOS groups. The latter demonstrated a two-fold increase in AMH over controls, similar to those found in previous studies $[27,28]$.

It has been suggested that AMH may be a useful diagnostic test for PCOS with cut-off thresholds ranging from $>2.8$ to $>8.16 \mathrm{ng} / \mathrm{mL}[29,30]$. As in prior studies, we found that AMH $>3.19 \mathrm{ng} / \mathrm{mL}$ was significantly associated with PCOS. The variations in AMH threshold may be attributed to many factors including ethnicity and analytical method variations [31]. The existence of such racial/ethnic differences in ovarian reserves, as reflected by $\mathrm{AMH}$, may be also attributed to various genetic factors and environmental factors such as obesity, smoking, and vitamin D deficiency, which have been shown to correlate with serum AMH levels [32].

Menarche age showed a significant association with AMH, as we found that PCOS patients experiencing menarche at an older age were more prone to have high AMH levels. While previous studies have concluded that women who experience early menarche have significantly higher AMH as young adults $[33,34]$, this was contrary to our findings. This could be attributed to the variation in the tested populations, as both studies were conducted among non-PCOS subjects.

Moreover, our results showed that women with PCOS with AMH $>3.19 \mathrm{ng} / \mathrm{mL}$ had a greater prevalence of PCOM and OA. A previous study proposed that women with AMH $>10 \mathrm{ng} / \mathrm{mL}$ have a greater prevalence of PCOS and OA [35]. Additionally, we have demonstrated that there is no association between $\mathrm{AMH}$ cut-off and $\mathrm{HA}$, while others have suggested $\mathrm{AMH}$ as a surrogate marker for HA [14].

The accuracy of AMH as a diagnostic criterion was previously examined in two groups by re-assessing the diagnosis of PCOS using Rotterdam criteria. They found that when PCOM in Rotterdam criteria was replaced by $\mathrm{AMH}$, any two of $\mathrm{AMH}$, OA or HA provided a sensitivity of $86.67 \%$ [28] or $83 \%$ [14]. After applying this classification to our cohort we achieved $78 \%$ sensitivity. Alternatively, when AMH was replaced by OA, $100 \%$ specificity and $81 \%$ sensitivity were obtained. Our results are similar to those in a previous study (92\% specificity and $79 \%$ sensitivity) [35]. Serum AMH levels in another recent study added values in classifying different PCOS phenotypes that might help clinicians identify patients at high risk of ovarian hyperstimulation syndrome and customize specific therapies [36].

\section{Conclusions}

There is a positive correlation between high serum AMH levels, PCOM, OA, age of menarche, and PCOS. AMH is a key diagnostic marker of ovarian dysfunction in PCOS patients in combination with other clinical features, especially in cases with ambiguous evaluation of PCOM via ultrasound. Although there is no single diagnostic cut-off for $\mathrm{AMH}$, it is still an objective and quantitative marker not affected by the day of menses. Future studies in different regions of Saudi Arabia should be undertaken to validate the cut-off.

Author Contributions: S.B. conceptualized the study. O.S.B. is an Obstetrician and Gynecologist who diagnosed the patients. N.A. and A.A.B. performed experiments and analyzed data. N.A. and S.B. wrote the manuscript. S.B. corrected the final version of the manuscript.

Funding: This work was supported by the Center of Innovation in Personalized Medicine (CIPM), King Abdulaziz University, Jeddah, Saudi Arabia.

Acknowledgments: The authors would like to show great appreciation to Najla'a Filmban for proofreading the manuscript, CIPM, King Abdulaziz University, Jeddah, Saudi Arabia; Angham Nawar (Radiology and Imaging technician at CIPM); and Jawaher Alsaedi (nurse at CIPM) for collecting the blood samples from the subjects. Additionally, the authors thank Salah Barnawi (Statistics Department, King Fahad Medical Research Center, King Abdulaziz University, Saudi Arabia) for his valuable advice on statistical methods.

Conflicts of Interest: The authors declare no conflict of interest. 


\section{References}

1. March, W.A.; Moore, V.M.; Willson, K.J.; Phillips, D.I.; Norman, R.J.; Davies, M.J. The prevalence of polycystic ovary syndrome in a community sample assessed under contrasting diagnostic criteria. Hum. Reprod. 2009, 25, 544-551. [CrossRef] [PubMed]

2. Rotterdam ESHRE/ASRM-Sponsored PCOS Consensus Workshop Group. Revised 2003 consensus on diagnostic criteria and long-term health risks related to polycystic ovary syndrome (PCOS). Hum. Reprod. 2004, 19, 41-47. [CrossRef] [PubMed]

3. Bustin, S.A.; Benes, V.; Garson, J.A.; Hellemans, J.; Huggett, J.; Kubista, M.; Mueller, R.; Nolan, T.; Pfaffl, M.W.; Shipley, G.L.; et al. The MIQE guidelines: Minimum information for publication of quantitative real-time PCR experiments. Clin. Chem. 2009, 55, 611-622. [CrossRef] [PubMed]

4. Kosus, N.; Kosus, A.; Turhan, N.O.; Kamalak, Z. Do threshold values of ovarian volume and follicle number for diagnosing polycystic ovarian syndrome in Turkish women differ from western countries? Eur. J. Obstet. Gynecol. Reprod. Biol. 2011, 154, 177-181. [CrossRef] [PubMed]

5. Chen, Y.; Li, L.; Chen, X.; Zhang, Q.; Wang, W.; Li, Y.; Yang, D. Ovarian volume and follicle number in the diagnosis of polycystic ovary syndrome in Chinese women. Ultrasound Obstet. Gynecol. 2008, 32, 700-703. [CrossRef]

6. Durlinger, A.; Visser, J.A.; Themmen, A. Regulation of ovarian function: The role of anti-Mullerian hormone. Reproduction 2002, 124, 601-609. [CrossRef]

7. Grynnerup, A.G.; Lindhard, A.; Sorensen, S. The role of anti-Mullerian hormone in female fertility and infertility-An overview. Acta Obstet. Gynecol. Scand. 2012, 91, 1252-1260. [CrossRef]

8. Laven, J.S.; Mulders, A.G.; Visser, J.A.; Themmen, A.P.; De Jong, F.H.; Fauser, B.C. Anti-Mullerian hormone serum concentrations in normoovulatory and anovulatory women of reproductive age. J. Clin. Endocrinol. Metab. 2004, 89, 318-323. [CrossRef]

9. Pigny, P.; Jonard, S.; Robert, Y.; Dewailly, D. Serum anti-Mullerian hormone as a surrogate for antral follicle count for definition of the polycystic ovary syndrome. J. Clin. Endocrinol. Metab. 2006, 91, 941-945. [CrossRef]

10. Bhide, P.; Gudi, A.; Shah, A.; Homburg, R. Serum anti-Mullerian hormone levels across different ethnic groups: A cross-sectional study. BJOG 2015, 122, 1625-1629. [CrossRef]

11. Nardo, L.G.; Yates, A.P.; Roberts, S.A.; Pemberton, P.; Laing, I. The relationships between AMH, androgens, insulin resistance and basal ovarian follicular status in non-obese subfertile women with and without polycystic ovary syndrome. Hum. Reprod. 2009, 24, 2917-2923. [CrossRef] [PubMed]

12. Dewailly, D.; Gronier, H.; Poncelet, E.; Robin, G.; Leroy, M.; Pigny, P.; Duhamel, A.; Catteau-Jonard, S. Diagnosis of polycystic ovary syndrome (PCOS): Revisiting the threshold values of follicle count on ultrasound and of the serum AMH level for the definition of polycystic ovaries. Hum. Reprod. 2011, 26, 3123-3129. [CrossRef] [PubMed]

13. Sahmay, S.; Aydin, Y.; Oncul, M.; Senturk, L.M. Diagnosis of Polycystic Ovary Syndrome: AMH in combination with clinical symptoms. J. Assist. Reprod. Genet. 2014, 31, 213-220. [CrossRef] [PubMed]

14. Dewailly, D.; Pigny, P.; Soudan, B.; Catteau-Jonard, S.; Decanter, C.; Poncelet, E.; Duhamel, A. Reconciling the definitions of polycystic ovary syndrome: The ovarian follicle number and serum anti-Mullerian hormone concentrations aggregate with the markers of hyperandrogenism. J. Clin. Endocrinol. Metab. 2010, 95, 4399-4405. [CrossRef]

15. Tata, B.; Mimouni, N.E.H.; Barbotin, A.L.; Malone, S.A.; Loyens, A.; Pigny, P.; Dewailly, D.; Catteau-Jonard, S.; Sundstrom-Poromaa, I.; Piltonen, T.T.; et al. Elevated prenatal anti-Mullerian hormone reprograms the fetus and induces polycystic ovary syndrome in adulthood. Nat. Med. 2018, 24, 834-846. [CrossRef]

16. Iglesias, C.; Banker, M.; Mahajan, N.; Herrero, L.; Meseguer, M.; Garcia-Velasco, J.A. Ethnicity as a determinant of ovarian reserve: Differences in ovarian aging between Spanish and Indian women. Fertil. Steril. 2014, 102, 244-249. [CrossRef]

17. Pigny, P.; Gorisse, E.; Ghulam, A.; Robin, G.; Catteau-Jonard, S.; Duhamel, A.; Dewailly, D. Comparative assessment of five serum antimullerian hormone assays for the diagnosis of polycystic ovary syndrome. Fertil. Steril. 2016, 105, 1063-1069. [CrossRef]

18. Song, D.K.; Oh, J.Y.; Lee, H.; Sung, Y.A. Differentiation between polycystic ovary syndrome and polycystic ovarian morphology by means of an anti-Mullerian hormone cutoff value. Korean J. Intern. Med. 2017, 32, 690-698. [CrossRef] 
19. Matsuzaki, T.; Munkhzaya, M.; Iwasa, T.; Tungalagsuvd, A.; Yano, K.; Mayila, Y.; Yanagihara, R.; Tokui, T.; Kato, T.; Kuwahara, A.; et al. Relationship between serum anti-Mullerian hormone and clinical parameters in polycystic ovary syndrome. Endocr. J. 2017, 64, 531-541. [CrossRef]

20. Batarfi, A.A.; Filimban, N.; Bajouh, O.S.; Dallol, A.; Chaudhary, A.G.; Bakhashab, S. MC4R variants rs12970134 and rs17782313 are associated with obese polycystic ovary syndrome patients in the Western region of Saudi Arabia. BMC Med. Genet. 2019, 20, 144. [CrossRef]

21. Teede, H.; Hutchison, S.; Zoungas, S.; Meyer, C. Insulin resistance, the metabolic syndrome, diabetes, and cardiovascular disease risk in women with PCOS. Endocrine 2006, 30, 45-53. [CrossRef]

22. Diamanti-Kandarakis, E.; Dunaif, A. Insulin resistance and the polycystic ovary syndrome revisited: An update on mechanisms and implications. Endocr. Rev. 2012, 33, 981-1030. [CrossRef] [PubMed]

23. Barthelmess, E.K.; Naz, R.K. Polycystic ovary syndrome: Current status and future perspective. Front. Biosci. 2014, 6, 104-119.

24. Piouka, A.; Farmakiotis, D.; Katsikis, I.; Macut, D.; Gerou, S.; Panidis, D. Anti-Mullerian hormone levels reflect severity of PCOS but are negatively influenced by obesity: Relationship with increased luteinizing hormone levels. Am. J. Physiol. Endocrinol. Metab. 2009, 296, E238-E243. [CrossRef]

25. Wiweko, B.; Maidarti, M.; Priangga, M.D.; Shafira, N.; Fernando, D.; Sumapraja, K.; Natadisastra, M.; Hestiantoro, A. Anti-mullerian hormone as a diagnostic and prognostic tool for PCOS patients. J. Assist. Reprod. Genet. 2014, 31, 1311-1316. [CrossRef]

26. La Marca, A.; Stabile, G.; Artenisio, A.C.; Volpe, A. Serum anti-Mullerian hormone throughout the human menstrual cycle. Hum. Reprod. 2006, 21, 3103-3107. [CrossRef]

27. Sathyapalan, T.; Al-Qaissi, A.; Kilpatrick, E.S.; Dargham, S.R.; Atkin, S.L. Anti-Mullerian hormone measurement for the diagnosis of polycystic ovary syndrome. Clin. Endocrinol. 2018, 88, 258-262. [CrossRef]

28. Saxena, U.; Ramani, M.; Singh, P. Role of AMH as Diagnostic Tool for Polycystic Ovarian Syndrome. J. Obstet. Gynaecol. India 2018, 68, 117-122. [CrossRef]

29. Yue, C.Y.; Lu, L.K.; Li, M.; Zhang, Q.L.; Ying, C.M. Threshold value of anti-Mullerian hormone for the diagnosis of polycystic ovary syndrome in Chinese women. PLoS ONE 2018, 13, e0203129. [CrossRef]

30. Eilertsen, T.B.; Vanky, E.; Carlsen, S.M. Anti-Mullerian hormone in the diagnosis of polycystic ovary syndrome: Can morphologic description be replaced? Hum. Reprod. 2012, 27, 2494-2502. [CrossRef]

31. Iliodromiti, S.; Kelsey, T.W.; Anderson, R.A.; Nelson, S.M. Can anti-Mullerian hormone predict the diagnosis of polycystic ovary syndrome? A systematic review and meta-analysis of extracted data. J. Clin. Endocrinol. Metab. 2013, 98, 3332-3340. [CrossRef] [PubMed]

32. Tal, R.; Seifer, D.B. Potential mechanisms for racial and ethnic differences in antimullerian hormone and ovarian reserve. Int. J. Endocrinol. 2013, 2013, 818912. [CrossRef] [PubMed]

33. Bragg, J.M.; Kuzawa, C.W.; Agustin, S.S.; Banerjee, M.N.; McDade, T.W. Age at menarche and parity are independently associated with Anti-Mullerian hormone, a marker of ovarian reserve, in Filipino young adult women. Am. J. Hum. Biol. 2012, 24, 739-745. [CrossRef] [PubMed]

34. Kerkhof, G.F.; Leunissen, R.W.; Willemsen, R.H.; de Jong, F.H.; Visser, J.A.; Laven, J.S.; Hokken-Koelega, A.C. Influence of preterm birth and small birth size on serum anti-Mullerian hormone levels in young adult women. Eur. J. Endocrinol. 2010, 163, 937-944. [CrossRef] [PubMed]

35. Tal, R.; Seifer, D.B.; Khanimov, M.; Malter, H.E.; Grazi, R.V.; Leader, B. Characterization of women with elevated antimullerian hormone levels $(\mathrm{AMH})$ : Correlation of $\mathrm{AMH}$ with polycystic ovarian syndrome phenotypes and assisted reproductive technology outcomes. Am. J. Obstet. Gynecol. 2014, 211. [CrossRef] [PubMed]

36. Cela, V.; Obino, M.E.R.; Alberga, Y.; Pinelli, S.; Sergiampietri, C.; Casarosa, E.; Simi, G.; Papini, F.; Artini, P.G. Ovarian response to controlled ovarian stimulation in women with different polycystic ovary syndrome phenotypes. Gynecol. Endocrinol. 2018, 34, 518-523. [CrossRef]

(C) 2019 by the authors. Licensee MDPI, Basel, Switzerland. This article is an open access article distributed under the terms and conditions of the Creative Commons Attribution (CC BY) license (http://creativecommons.org/licenses/by/4.0/). 